Supporting Information for

\title{
Angiogenesis Promotion by Combined Administration of \\ DFO and Vein Endothelial Cells using Injectable, Biodegradable, Nanocomposite Hydrogel Scaffolds
}

Kimika Ono, Manami Sumiya, Naohiro Yoshinobu, Tatsuya Dode, Tokitaka Katayama, Natsumi Ueda, and Koji Nagahama*

Department of Nanobiochemistry, Frontiers of Innovative Research in Science and Technology (FIRST), Konan University, 7-1-20 Minatojima-Minamimachi, Chuo-ku, Kobe 650-0047, Japan

E-mail of the corresponding author: nagahama@konan-u.ac.jp 

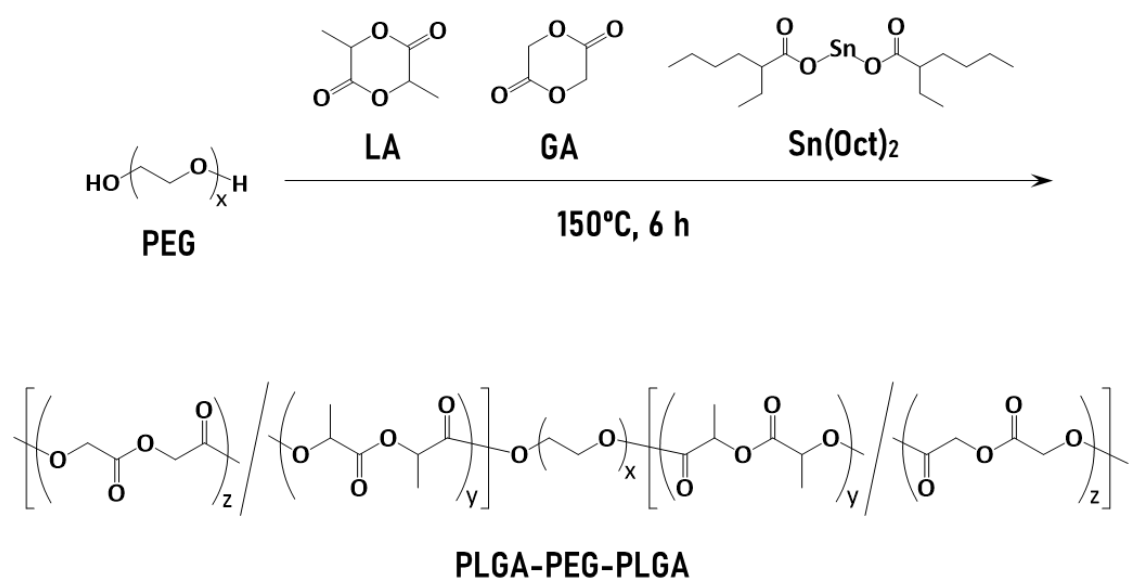

Scheme S1. Synthesis of PLGA-PEG-PLGA copolymers. 


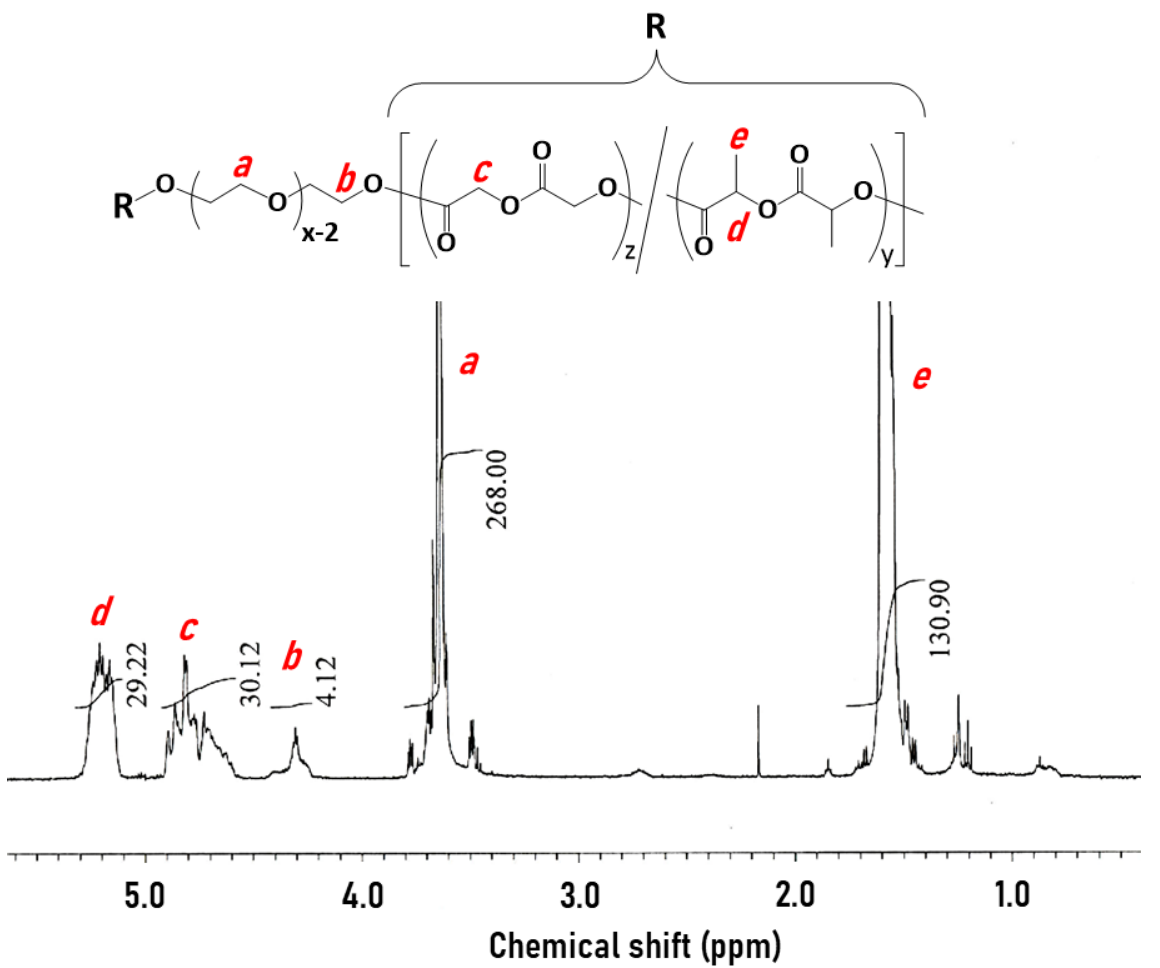

Figure S1. ${ }^{1} \mathrm{H}-\mathrm{NMR}$ spectrum of the synthesized PLGA-PEG-PLGA copolymer. 
Table S1. Characterization of PLGA-PEG-PLGA copolymers used in this study.

\begin{tabular}{|c|c|c|c|c|c|}
\hline copolymer & DP of LA & DP of GA & $M_{\mathrm{w}}$ of PEG & $M_{\mathrm{w}}$ of copolymer & $M_{\mathrm{w}} / M_{\mathrm{n}}^{c}$ \\
\hline PLGA-PEG-PLGA & 8 & 4 & 3,000 & 6,300 & 1.15 \\
\hline
\end{tabular}

${ }^{a}$ Estimated by ${ }^{1} \mathrm{H}-\mathrm{NMR} .{ }^{b}$ Estimated by following equation; $M_{\mathrm{w}}$ of copolymer $=M_{\mathrm{w}}$ of PEG segment $+2 \times\left(M_{\mathrm{w}}\right.$ of PLGA segment). ${ }^{c}$ Estimated by GPC (eluent: DMSO, standard: PEG). 

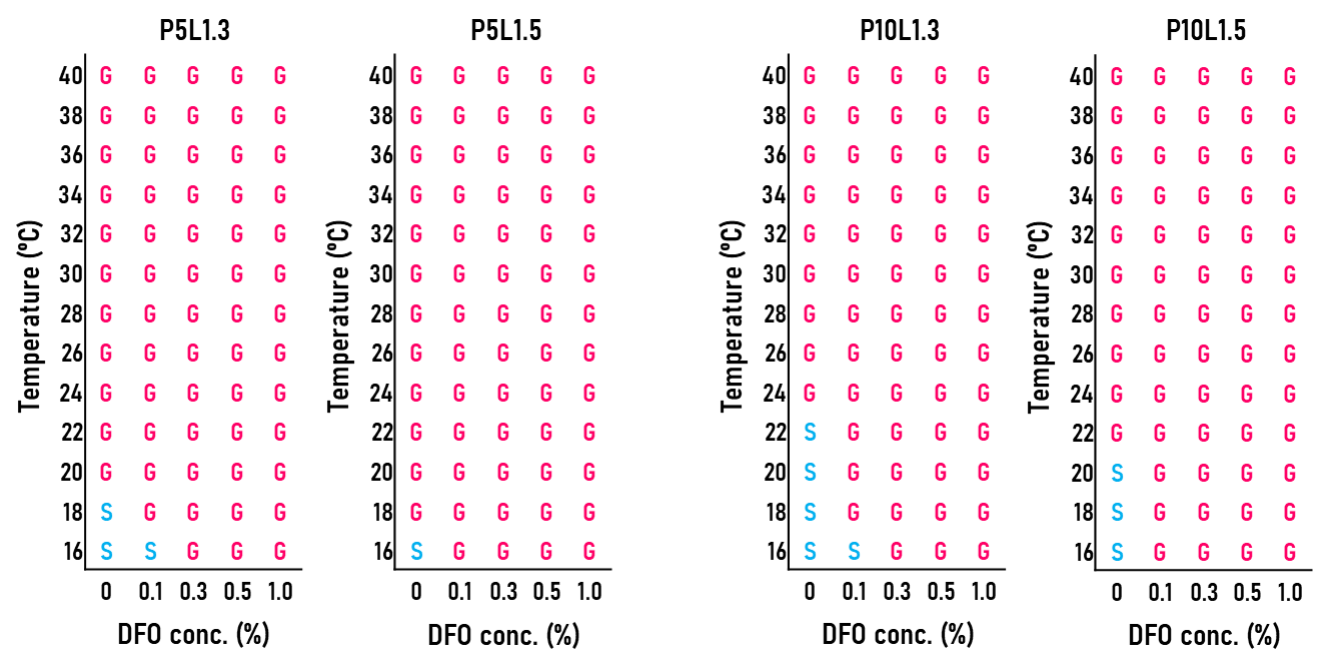

Figure S2. Phase diagrams of DFO-laden PLGA-PEG-PLGA/LAPONITE nanocomposite gels with different composition ratios. 


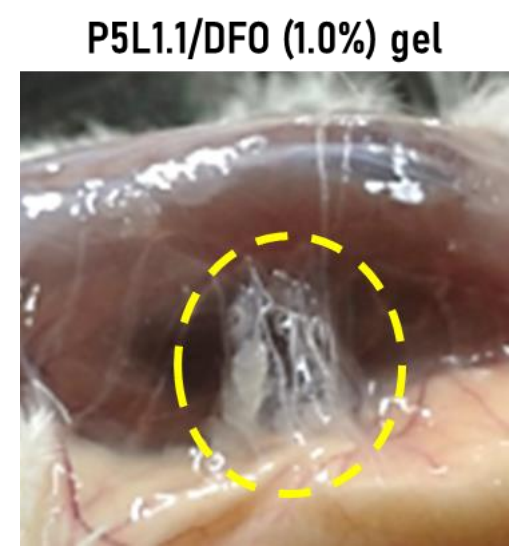

Figure S3. Photograph of DFO $(1.0 \%)$ and HUVEC $\left(1.0 \times 10^{6}\right)$-loaded P5L1.1 gel formed in subcutaneous tissue after injection. 